\title{
Spin precession in spin-orbit coupled weak links: Coulomb repulsion and Pauli quenching
}

\author{
R. I. Shekhter, ${ }^{1}$ O. Entin-Wohlman, ${ }^{2,3, \text { | } M . ~ J o n s o n, ~}{ }^{1}$ and A. Aharony ${ }^{2,3}$ \\ ${ }^{1}$ Department of Physics, University of Gothenburg, SE-412 96 Göteborg, Sweden \\ ${ }^{2}$ Raymond and Beverly Sackler School of Physics and Astronomy, Tel Aviv University, Tel Aviv 69978, Israel \\ ${ }^{3}$ Physics Department, Ben Gurion University, Beer Sheva 84105, Israel
}

(Dated: July 15, 2021)

\begin{abstract}
A simple model for the transmission of pairs of electrons through a weak electric link in the form of a nanowire made of a material with strong electron spin-orbit interaction (SOI) is presented, with emphasis on the effects of Coulomb interactions and the Pauli exclusion principle. The constraints due to the Pauli principle are shown to "quench" the coherent SOI-induced precession of the spins when the spatial wave packets of the two electrons overlap significantly. The quenching, which results from the projection of the pair's spin states onto spin-up and spin-down states on the link, breaks up the coherent propagation in the link into a sequence of coherent hops that add incoherently. Applying the model to the transmission of Cooper pairs between two superconductors, we find that in spite of Pauli quenching, the Josephson current oscillates with the strength of the SOI, but may even change its sign (compared to the limit of Coulomb blockade, when the quenching is absent). Conditions for an experimental detection of these features are discussed.
\end{abstract}

PACS numbers: $72.25 . \mathrm{Hg}, 72.25 . \mathrm{Rb}$

Introduction. Electronic devices comprising weak electric links made of a material with strong electronic spinorbit interaction (SOI) are promising candidates for spintronics applications and offer new modes of operation. This follows from the possibility to manipulate the spin structure of electrons flowing through the link via the effect of external fields on the SOI. Transport experiments on electrons in gated semiconductor heterostructures demonstrated that the strength of the Rashba $a^{112}$ SOI can be both significant and controlled by gate voltages. $\frac{3}{5}[5$ Theoretically, it was proposed that nanowire weak links made of SOI-active materials suspended between bulk electrodes can act as "Rashba spin-splitters" and lead to mechanically controlled spin-selective transport ${ }^{\underline{6}}$ and spintro-voltaic effects ${ }^{77}$ These predictions can be understood within a semiclassical picture: as the electrons pass through the link, their spins precess around an effective magnetic field associated with the SOI. This spin dynamics splits the electron wave function into different spin states and leads to a certain probability, which can be controlled externally, for the spins to be flipped as they emerge from the link ${ }^{8}$

The spin-splitting phenomenon becomes more complicated for the transmission of a pair of electrons through an SOI-active link. Weak superconductivity, established by the transfer of Cooper pairs through a nonsuperconducting material, is the obvious system for studying spin-splitting in the transmission of electron pairs; it is analyzed in this paper. Other examples of pairs tunneling appear in non-superconducting transport involving higher-order tunneling events: shot noise in weak electric links ${ }^{\sqrt{9}}$ and cross-correlations of currents in multi-terminal mesoscopic structures 10

Since two electrons in the same spin state cannot occupy the same place simultaneously (Pauli principle), possible effects due to the Coulomb blockade of electron tunneling and the constraints on the spin dynamics have to be considered. A fundamental question is whether these constraints destroy the coherent spin precession of individual electrons and destroy the spin-splitting phenomenon. Here we estimate the outcome of these effects. As a motivation for the structure of our model, we propose a semiclassical picture of the transfer of a pair of electrons through the SOI-active weak link. The two electrons tunnel one by one at separate times, say $t_{1}<t_{2}$, from the source electrode to the nanowire. Once there, they propagate as wave packets with different velocities until leaving the nanowire by tunneling into the drain electrode, also at different times, $t_{3}<t_{4}$. If the first electron to enter leaves before the second comes in, i.e., $t_{1}<t_{3}<t_{2}<t_{4}$, then there is only one electron in the wire at any given time. This case is termed "singleelectron", or "s-channel" - transmission (though, importantly, the transmission is that of a pair of electrons that traverse the link one by one). Effects due to the Coulomb interaction and the Pauli principle on the motion are ignored; the spatial motion is that of free electrons and the spin dynamics evolve coherently according to the SOI on the link.

If, on the other hand, the second electron tunnels into the nanowire before the first one has left, i.e., $t_{1}<t_{2}<$ $t_{3}<t_{4}$, then there are two electrons in the nanowire during the time interval $\left\{t_{2}, t_{3}\right\}$. This case is called "doubleelectron", or "d-channel" - transmission. Because of the different longitudinal velocities of their wave packets, the two electrons may or may not meet somewhere on the wire. If they do, then the constraints due to the Pauli principle need to be considered. This is accomplished in our model by assuming that the two electrons meet at a point modeled by a quantum dot with two spin states ("up" and "down")!11 The Pauli principle is assumed to be effective only there; it is taken into account by pro- 
jecting the spin state of one electron on the spin-up state of the dot and that of the other on the spin-down state there. The projection breaks the coherent evolution of the spin states, which we refer to as "Pauli quenching" of the spin precession. As the electrons leave the meeting point their spin states again evolve coherently. In this sense the coherent propagation of the electrons through the link is broken up into two pair-hopping events. The Coulomb interaction in the d-channel is accounted for only at the meeting point. Assuming that the electrons are equally likely to meet anywhere along the nanowire, the outcome of this event for a particular choice of meeting location is averaged over all possible choices.

Below, we first introduce the Hamiltonian of our model and detail the calculation of the transmission of Cooper pairs between two superconductors connected by an SOIactive weak link. Next, the spin-precession factor of each of the two processes is presented, and the way the disparity between the two reflects the coherence of the s-channel process, and the incoherence of the d-channel one is explained. Explicit expressions for these factors, for a specific model of the Rashba linear SOI, are then analyzed, followed by a discussion of relevant experiments.

The model and the current. As mentioned, the "meeting point" of the two electrons is represented by a single-level quantum dot of energy $\epsilon$, that can accommodate the transferred electrons in "up" and "down" spin states. The passage of the electrons in and out of the dot is viewed as single-electron tunneling events, whose amplitudes include the electronic spin precession; the reservoirs that supply the electrons are two bulk BCS superconductors, coupled together by a nanowire on which the quantum dot is located. Thus, the Hamiltonian of the entire junction reads

$$
\mathcal{H}=\mathcal{H}_{0}+\mathcal{H}_{\text {tun }},
$$

where $\mathcal{H}_{0}$ describes the decoupled system, the Hamiltonian of the quantum dot and that of the leads,

$$
\mathcal{H}_{0}=\sum_{\sigma} \epsilon d_{\sigma}^{\dagger} d_{\sigma}+U d_{\uparrow}^{\dagger} d_{\uparrow} d_{\downarrow}^{\dagger} d_{\downarrow}+\sum_{\alpha=L, R} \mathcal{H}_{\text {lead }}^{\alpha} .
$$

Here, $d_{\sigma}\left(d_{\sigma}^{\dagger}\right)$ annihilates (creates) an electron in the spin state $|\sigma\rangle$ on the dot and $U$ denotes the Coulomb repulsion energy. The BCS leads are described by the annihilation (creation) operators of the electrons there, $c_{\mathbf{k}(\mathbf{p}) \sigma}$ $\left(c_{\mathbf{k}(\mathbf{p}) \sigma}^{\dagger}\right) . \quad[\mathbf{k}(\mathbf{p})$ enumerates the single-particle orbital states on the left (right) lead.] Denoting by $\epsilon_{k(p)}$ the single-electron energy measured relative to the chemical potential $\frac{122}{12}$ the Hamiltonian of the leads is

$$
\begin{aligned}
& \mathcal{H}_{\text {lead }}^{\alpha=L(R)}=\sum_{\mathbf{k}(\mathbf{p}), \sigma} \epsilon_{k(p)} c_{\mathbf{k}(\mathbf{p}) \sigma}^{\dagger} c_{\mathbf{k}(\mathbf{p}) \sigma} \\
& -\Delta_{L(R)} \sum_{\mathbf{k}(\mathbf{p})}\left(e^{\left.i \phi_{L(R)}\right)} c_{\mathbf{k}(\mathbf{p}) \uparrow}^{\dagger} c_{-\mathbf{k}(-\mathbf{p}) \downarrow}^{\dagger}+\text { H.c. }\right),
\end{aligned}
$$

where $\Delta_{L(R)}$ and $\phi_{L(R)}$ are the amplitude and the phase of the superconducting order parameters.
The tunneling Hamiltonian is the key component of our model,

$$
\mathcal{H}_{\text {tun }}=\mathcal{H}_{L D}+\mathcal{H}_{R D}+\mathcal{H}_{D L}+\mathcal{H}_{D R}
$$

The transfer of an electron from the spin state $\left|\sigma^{\prime}\right\rangle$ on the dot to the state $|\mathbf{k}(\mathbf{p}), \sigma\rangle$ in the left (right) reservoir is expressed by

$$
\mathcal{H}_{L(R) D}=\sum_{\mathbf{k}(\mathbf{p}), \sigma, \sigma^{\prime}}\left[t_{\mathbf{k}(\mathbf{p})}\right]_{\sigma \sigma^{\prime}} c_{\mathbf{k}(\mathbf{p}) \sigma}^{\dagger} d_{\sigma^{\prime}}
$$

while the reverse process, from the state $|\mathbf{k}(\mathbf{p}), \sigma\rangle$ in the left (right) lead to the spin state $\left|\sigma^{\prime}\right\rangle$ on the dot, is $\mathcal{H}_{D L(R)}=\left[\mathcal{H}_{L(R) D}\right]^{\dagger}$. The amplitude $\left[t_{\mathbf{k}(\mathbf{p})}\right]_{\sigma \sigma^{\prime}}$ allows for spin flips during the tunneling. It is conveniently separated into a (scalar) orbital amplitude, and a matrix that contains the effects of the SOI (whether of the Rashba $a^{112}$ or the Dresselhaus ${ }^{13}$ type), and also the dependence on the spatial direction of the SOI-active wire. For the linear $\mathrm{SOl}$

$$
\mathbf{t}_{\mathbf{k}}^{L D}=i t_{L} e^{-i k_{\mathrm{F}} d_{L}} \mathbf{W}^{L D},
$$

where $k_{\mathrm{F}}$ is the Fermi wave vector in the leads, and $d_{L}$ is the length of the bond between the left lead and the dot. The superscript indicates the tunneling direction, from the dot to the left lead, etc. Specific forms for $\mathbf{W}$ are discussed below.

Since the two electrons of a Cooper pair are in timereversed states we also have to consider the transfer of an electron from the time-reversed spin state $\left|\bar{\sigma}^{\prime}\right\rangle \equiv\left(i \sigma_{y}\right)\left|\sigma^{\prime}\right\rangle$ on the dot to the state $|-\mathbf{k}(-\mathbf{p}), \bar{\sigma}\rangle$ in the left (right) lead. The amplitude for this process is $\left[\bar{t}_{\mathbf{k}(\mathbf{p})}\right]_{\overline{\sigma \sigma^{\prime}}}$, where

$$
\overline{\mathbf{t}}_{\mathbf{k}(\mathbf{p})}^{L(R) D} \equiv \hat{\mathbf{T}} \mathbf{t}_{\mathbf{k}(\mathbf{p})}^{L(R)} \hat{\mathbf{T}}^{-1} ; \quad \hat{\mathbf{T}}=K\left(i \sigma_{y}\right),
$$

( $\sigma_{y}$ is a Pauli matrix, and $K$ is the complex conjugation operator). We note here the important relation

$$
\left[\bar{t}_{\mathbf{k}(\mathbf{p})}^{D L(R)}\right]_{\overline{\sigma \sigma^{\prime}}}=\left[t_{\mathbf{k}(\mathbf{p})}^{D L(R) *}\right]_{\sigma \sigma^{\prime}},
$$

used below to eliminate the time-reversed tunneling amplitudes from our final results.

The flow of electrons between the two superconductors is analyzed by studying the equilibrium Josephson current, i.e., the rate by which electrons leave the left superconductor $^{15}$ (we use $\hbar=1$ )

$$
J_{L}=-e(d / d t)\left\langle\sum_{\mathbf{k}, \sigma} c_{\mathbf{k} \sigma}^{\dagger} c_{\mathbf{k} \sigma}\right\rangle=-2 e \operatorname{Im}\left\langle\mathcal{H}_{L D}(t)\right\rangle
$$

where the angular brackets denote quantum averaging. $J_{L}$ is evaluated using the $S$-matrix, $\left\langle\mathcal{H}_{L D}(t)\right\rangle=\left\langle S^{-1}(t,-\infty) \mathcal{H}_{L D}(t) S(t,-\infty)\right\rangle$, with $\mathcal{H}_{L D}(t)=\exp \left[i \mathcal{H}_{0} t\right] \mathcal{H}_{L D} \exp \left[-i \mathcal{H}_{0} t\right]$, and the quantum average is with respect to $\mathcal{H}_{0}$. As it is at least fourthorder in the tunneling Hamiltonian, it is found from the expansion up to third order of the $S$-matrix ${ }^{[16}$ The 
energy level on the dot is assumed to lie well above the chemical potential of the leads, and thus the small parameter of the expansion is $\Gamma / \epsilon$, where $\Gamma=\Gamma_{L}+\Gamma_{R}$ is the width of the resonance level created on the dot due to the coupling with the bulk reservoirs. This implies that the perturbation expansion is carried out on a dot which is initially empty 17

The lowest-order current results from the processes in which two electrons are injected into and extracted from the dot. Two groups of terms can be identified. In the first double occupancy on the dot does not occur, and the transfer of the electron pair is accomplished by a sequential tunneling of the paired electrons one by one. These terms, which form the s-channel, contain $\left\langle d_{\sigma_{i}}\left(t_{i}\right) d_{\sigma_{j}}^{\dagger}\left(t_{j}\right) d_{\sigma_{i}^{\prime}}\left(t_{i^{\prime}}\right) d_{\sigma_{j}^{\prime}}^{\dagger}\left(t_{j^{\prime}}\right)\right\rangle$. The Pauli exclusion principle is not active here; the spin states are determined such that the initial and final states of the dot are empty (i.e., $\sigma_{i}^{\prime}=\sigma_{j}^{\prime}$ and $\sigma_{i}=\sigma_{j}$ ). The contribution to the Josephson current from these is 18

$$
J^{\mathrm{s}}=I_{0} F^{\mathrm{s}}(\epsilon / \Delta) \mathcal{A}^{\mathrm{s}},
$$

where $\Delta_{L}=\Delta_{R}=\Delta$ is assumed. For a short link the prefactor is ${ }^{19]} I_{0}=2 e\left[\Gamma_{L} \Gamma_{R} / \Delta\right] \sin \left(\phi_{R}-\phi_{L}\right)$, and

$$
\begin{aligned}
F^{\mathrm{s}}(\widetilde{\epsilon}) & =\int_{-\infty}^{\infty}\left(d \zeta_{k} / \pi\right) \int_{-\infty}^{\infty}\left(d \zeta_{p} / \pi\right)\left[\left(\cosh \zeta_{k}+\widetilde{\epsilon}\right)\right. \\
& \left.\times\left(\cosh \zeta_{k}+\cosh \zeta_{p}\right)\left(\cosh \zeta_{p}+\widetilde{\epsilon}\right)\right]^{-1} .
\end{aligned}
$$

The spin-precession factor $\mathcal{A}^{\mathrm{s}}$ in Eq. (10) is given in Eq. 15 and discussed below. In the second group of terms the dot is doubly occupied during the tunneling; these terms, of the generic form $\left\langle d_{\sigma_{i}}\left(t_{i}\right) d_{\sigma_{j}}\left(t_{j}\right) d_{\sigma_{i}^{\prime}}^{\dagger}\left(t_{i^{\prime}}\right) d_{\sigma_{j}^{\prime}}^{\dagger}\left(t_{j^{\prime}}\right)\right\rangle$, constitute the d-channel. The Pauli exclusion-principle constrains the spin states on the dot, $\sigma_{i}^{\prime}=-\sigma_{j}^{\prime}$ and $\sigma_{i}=-\sigma_{j}$. They contribute

$$
J^{\mathrm{d}}=I_{0} F^{\mathrm{d}}(\epsilon / \Delta, U / \Delta) \mathcal{A}^{\mathrm{d}},
$$

where $\mathcal{A}^{d}$ is the spin precession factor of these processes [Eq. [16] ] and

$$
\begin{aligned}
F^{\mathrm{d}}(\widetilde{\epsilon}, \widetilde{U}) & \left.=\int_{-\infty}^{\infty}\left(d \zeta_{k} / \pi\right) \int_{-\infty}^{\infty} d \zeta_{p} / \pi\right)\left[\left(\cosh \zeta_{k}+\widetilde{\epsilon}\right)\right. \\
& \left.\times(2 \widetilde{\epsilon}+\widetilde{U})\left(\cosh \zeta_{p}+\widetilde{\epsilon}\right)\right]^{-1} .
\end{aligned}
$$

Both $\mathcal{A}^{\mathrm{s}}=1$ and $\mathcal{A}^{\mathrm{d}}=1$ in the absence of the SOI.

The complete Josephson current is the sum of the contributions from the two types of processes, $J=J^{s}+J^{d}$,

$$
J=J_{0} \frac{\mathcal{A}^{\mathrm{s}} F^{\mathrm{s}}(\epsilon / \Delta)+2 \mathcal{A}^{\mathrm{d}} F^{\mathrm{d}}(\epsilon / \Delta, U / \Delta)}{F^{\mathrm{s}}(\epsilon / \Delta)+2 F^{\mathrm{d}}(\epsilon / \Delta, U / \Delta)},
$$

where $J_{0}=I_{0}\left[F^{\mathrm{s}}(\epsilon / \Delta)+2 F^{\mathrm{d}}(\epsilon / \Delta, U / \Delta)\right]$ is the equilibrium Josephson current of a junction with no SOI. Note that $J$ depends on the location of the dot, via the spinprecession factors $\mathcal{A}^{\mathrm{s}}$ and $\mathcal{A}^{\mathrm{d} ! 20}$ The Coulomb repulsion on the dot, $U$, affects the relative weights of the contributions from the s- and the d-channels: although $F^{\mathrm{s}}$ and $F^{\mathrm{d}}$ are independent of the SOI strength, they weigh differently the spin-precession factors. This competition modifies significantly the current.

The spin precession. In terms of the SOI amplitudes, Eq. (6), and using the symmetry relation Eq. (8), the spin-precession factor $\mathcal{A}^{\mathrm{s}}$ of the s-channel is 21

$$
\mathcal{A}^{\mathrm{s}}=\left|W_{\uparrow \uparrow}^{L R}\right|^{2}-\left|W_{\uparrow \downarrow}^{L R}\right|^{2} .
$$

$W_{\sigma_{L} \sigma_{R}}^{L R} \equiv \sum_{\sigma} W_{\sigma_{L} \sigma}^{L D} W_{\sigma \sigma_{R}}^{D R}$ is the direct tunneling amplitude between the leads. ${ }^{[22}$ As opposed, the spin precession factor of the d-channel cannot be expressed in terms of the direct amplitudes. Using Eqs. (6) and (8) we find

$$
\mathcal{A}^{\mathrm{d}}=\left(\left|W_{\uparrow \uparrow}^{L D}\right|^{2}-\left|W_{\uparrow \downarrow}^{L D}\right|^{2}\right)\left(\left|W_{\uparrow \uparrow}^{D R}\right|^{2}-\left|W_{\uparrow \downarrow}^{D R}\right|^{2}\right) .
$$

It is interesting to compare the structure of Eqs. 15) and (16) ${ }^{23}$ One notes that $\mathcal{A}^{\mathrm{d}}$ is a product of two factors of the same structure as the single factor in $\mathcal{A}^{\mathrm{s}}$. Our interpretation is that $\mathcal{A}^{\mathrm{s}}$ describes the coherent transfer of a Cooper pair from the right to the left lead, while $\mathcal{A}^{\mathrm{d}}$ describes first a coherent Cooper pair transfer from the right lead to the dot, where coherence is lost, then a second coherent transfer from the dot to the left lead.

Linear spin-orbit couplings. Though it is possible to calculate an effective SOI $a b$ initio, it is convenient to adopt the phenomenological Rashba Hamiltonian, ${ }^{2}$ valid for systems with a single high-symmetry axis that lack spatial inversion symmetry. For an electron of an effective mass $m^{*}$ and momentum $\mathbf{p}$ propagating along a wire where the SOI is active, it reads $\mathcal{H}_{\mathrm{so}}=\left(\hbar k_{\mathrm{so}} / m^{*}\right) \boldsymbol{\sigma} \cdot(\mathbf{p} \times$ $\hat{\mathbf{n}})$, where $\hat{\mathbf{n}}$ is a unit vector along the symmetry axis (the $\hat{\mathbf{c}}$-axis in hexagonal wurtzite crystals, the growth direction in a semiconductor heterostructure, the direction of an external electric field), and $k_{\mathrm{so}}$ is the strength of the SOI in units of inverse length. Using this Hamiltonian, we find $\sqrt{6}$

$$
\mathbf{t}_{\mathbf{k}(\mathbf{p})}=i t_{L} e^{i k_{\mathrm{F}} d_{L(R)}} \exp \left[i k_{\mathrm{so}} \mathbf{d}_{L(R)} \times \hat{\mathbf{n}} \cdot \boldsymbol{\sigma}\right],
$$

where $\mathbf{d}_{L(R)}$ is the radius vector pointing from the dot to the left (right) reservoir along the wire. The linear Dresselhaus SOl ${ }^{13}$ leads to a similar form.24/25

As an explicit example, we consider a straight nanowire ${ }^{26}$ of length $d$ as a weak link, on which the electrons are subjected to the Rashba SOI. The wire lies along $\hat{\mathbf{x}}$ in the $X Y$ plane, and is perpendicular to $\hat{\mathbf{n}}$. Then

$$
\mathcal{A}^{\mathrm{s}}=\cos \left(2 k_{\mathrm{so}} d\right), \mathcal{A}^{\mathrm{d}}=\cos \left(2 k_{\mathrm{so}} d_{L}\right) \cos \left(2 k_{\mathrm{so}} d_{R}\right),
$$

which reflects the structure of Eqs. (15) and 16). The spin-precession of the s-channel is independent of the position of the dot. Placing the dot at a distance $x$ from the left reservoir, the spin-precession of the d-channel is $\mathcal{A}^{\mathrm{d}}=\cos \left(2 k_{\mathrm{so}} d\right)+\sin \left[2 k_{\mathrm{so}}(d-x)\right] \sin \left(2 k_{\mathrm{so}} x\right)$. Averaging 


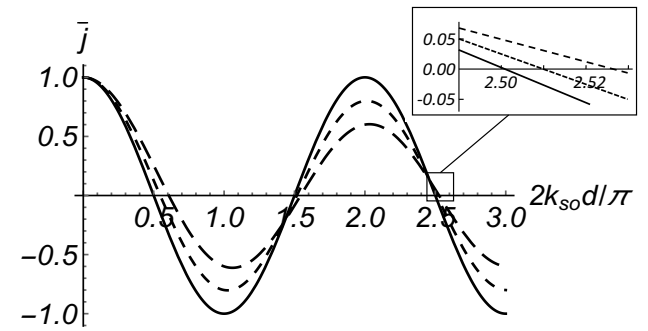

FIG. 1: The average Josephson current $\bar{j}$ vs. $2 k_{\text {so }} d / \pi$, for various values of the Coulomb repulsion, as encoded in $Z$ : $Z=0$ (full line) and $Z=0.2,0.4$ (increasing dashes). Inset: a narrower range, where $\bar{j}$ changes sign.

the normalized current over $x$ yields $\$ 11$

$$
\begin{aligned}
& \bar{j}=\int_{0}^{d} \frac{d x}{d} \frac{J(x)}{J_{0}}=\left[(1-Z) \cos \left(2 k_{\mathrm{so}} d\right)+Z \frac{\sin \left(2 k_{\mathrm{so}} d\right)}{2 k_{\mathrm{so}} d}\right], \\
& Z(U) \equiv \mathcal{F}^{\mathrm{d}}\left(\frac{\epsilon}{\Delta}, \frac{U}{\Delta}\right) /\left[\mathcal{F}^{\mathrm{s}}\left(\frac{\epsilon}{\Delta}\right)+2 \mathcal{F}^{\mathrm{d}}\left(\frac{\epsilon}{\Delta}, \frac{U}{\Delta}\right)\right] .
\end{aligned}
$$

In spite of the averaging over the possible locations where the two electrons' wave packets overlap considerably, the spin-orbit dynamics is preserved: the current oscillates as a function of the gate voltage which dictates $k_{\text {so }}$. Figure 1 shows $\bar{j}$ vs. $2 k_{\mathrm{so}} d$ for a range of values taken from the experiments, for several values of $Z$. In the Coulomb-blockade limit $U=\infty$ and $Z=0$ : the current exhibits the simple oscillations $\bar{j}=\cos \left(2 k_{\mathrm{so}} d\right)$. As $U$ decreases, $Z$ increases monotonically (for fixed $\epsilon / \Delta$ ) but remains smaller than $1 / 2$. It also decreases with increasing $\epsilon / \Delta$. At large $2 k_{\mathrm{so}} d \bar{j} \approx(1-Z) \cos \left(2 k_{\mathrm{so}} d\right)$; the Coulomb interaction reduces the magnitude of $\bar{j}$, but does not change its sign. For intermediate values of $2 k_{\mathrm{so}} d$ there appear small segments of $2 k_{\mathrm{so}} d$ in which the sign of the current also changes for $Z>0$, i.e., for $\tan \left(2 k_{\mathrm{so}} d\right) /\left(2 k_{\mathrm{so}} d\right)<(Z-1) / Z$. The inset in Fig. 1 zooms in on such a range; it broadens as $Z$ increases.

The capability to tune the SOI electrostatically by gate voltages was demonstrated on the inversion layer of a $\operatorname{In}_{0.75} \mathrm{Ga}_{0.25} \mathrm{As} / \mathrm{In}_{0.75} \mathrm{Al}_{0.25} \mathrm{As}$ semiconductor heterostructure. ${ }^{4}$ The spin-orbit coupling constant $\alpha$, mainly attributed to the Rashba SOI parameter $\alpha_{R}$, varied with gate voltage between roughly 150 and 300 meVÅ. Using $k_{\mathrm{so}}=m^{*} \alpha_{R} / \hbar^{2}$ and the measured $m^{*}=$ $0.041 \mathrm{~m}$ ( $\mathrm{m}$ is the mass of a free electron) one concludes that if a weak link were to be electrostatically defined in this system the argument $2 k_{\mathrm{so}} d$ of the trigonometric functions in Eq. (19) for a $1 \mu \mathrm{m}$ long link could be varied from $\sim 16$ to $\sim 32$. This amounts to a tuning over a range that is more than twice the period $2 \pi$ of these functions. A more recent experimental evidence for the SOI tunability is found in a dual gated InAs/GaSb quantum well where the Rashba SOI parameter $\alpha_{R}$ could be varied between 53 and $75 \mathrm{meV} \AA$, while the Dresselhaus
SOI was kept constant $\left[5\right.$ The stated value of $m^{*}=0.04 m$ implies that $2 k_{\mathrm{so}} d$ could be varied between $\sim 6$ to $\sim 8$, that is over about a third of the period $2 \pi$ if $d=1 \mu \mathrm{m}$.

The magnitude of the Josephson current through a quantum dot is set by the functions $\mathcal{F}^{\mathrm{s}}$ [Eq. [11] ] and $\mathcal{F}^{\mathrm{d}}$ [Eq. 13]], that are derived for short weak links. $\frac{19}{19}$ However, whereas the restriction on the length $d$ of the link might be strict, $d \ll \xi$, for the orbital part ( $\xi$ is the superconducting coherence length), it is far weaker for the spin-dependent part: $k_{\mathrm{so}} d \ll k_{\mathrm{F}} \xi$, since the spinprecession factors $\mathcal{A}^{\mathrm{s}}$ and $\mathcal{A}^{\mathrm{d}}$ are not sensitive to the energy dependence of the transmission amplitude. ${ }^{14}$

Summary. We have considered the spin splitting of Cooper pairs that carry a supercurrent through a weaklink Josephson junction. Our main result, expressed in Eq. 19p and shown in Fig. 1, is that Coulomb repulsion and Pauli quenching do affect the current, but do not destroy the possibility to tune it by changing the strength of the spin-orbit interaction.

The oscillatory dependence of the supercurrent on the SOI strength results from a rather complex interference between different transmission events: the single-electron transmission one (s-channel), that yields $J^{\mathrm{s}}$, Eq. 10, and the double-electron transmission (d-channel) that gives $J^{\text {d }}$, Eq. $(12)$. In the s-channel the two electrons are transferred one by one, so that at any time during the tunneling there is only one electron in the link. By contrast, in the d-channel both electrons appear in the link for some period of time, which means that in the Coulomb blockade limit the transfer of Cooper pairs in this channel is completely suppressed. The s-channel has two coherent transmission channels, one where the spins of both members of the pair are preserved [first term in Eq. [15)] and one where they are both flipped (second term there). This double spin reversal is equivalent to a permutation of the paired electrons, which explains the difference in sign between the two terms. As the Coulomb blockade is lifted, the probability of pairs to be transferred in the d-channel increases. As seen from Eq. 16) for the spin precession factor $\mathcal{A}^{\mathrm{d}}$, the d-channel involves coherent transfers of the pairs separated by a Pauli quenching that breaks coherence. Remarkably enough, each of the two factors of $\mathcal{A}^{\mathrm{d}}$, which describes two coherent "hops", have the same structure as the result $\mathcal{A}^{\mathrm{s}}$.

The pronounced oscillations of the supercurrent and the sign reversal can be observed for plausible lengths of the weak link, of the order of a micron, supposedly achievable by suitably-designed geometries of the gates. This result indicates interesting phenomena caused by SOI-induced spin polarization of Cooper pairs.

The model, suggested here, allows for a unified approach to treat an interplay between spin- and charge related phenomena, which are in the center of nowadays nanoelectronics. An immediate generalization of this approach would be important for exploring new device functionalities such as spin-orbit controlled shot noise in nanostructures and spin-orbit effect in current crosscorrelations in multi-terminal nanodevices. 
We thank the Computational Science Research Center in Beijing for the hospitality that allowed for the accomplishment of this project. RIS and MJ thank the IBS Center for Theoretical Physics of Complex Systems, Daejeon, Rep. of Korea, and OEW and AA thank the Dept. of Physics, Univ. of Gothenburg, for hospitality. This work was partially supported by the Swedish Research Council (VR), by the Israel Science Foundation (ISF) and by the infrastructure program of Israel Ministry of Science and Technology under contract 3-11173.
* Electronic address: oraentin@bgu.ac.il

1 E. I. Rashba, Properties of semiconductors with an extremum loop .1. Cyclotron and combinational resonance in a magnetic field perpendicular to the plane of the loop, Fiz. Tverd. Tela (Leningrad) 2, 1224 (1960) [Sov. Phys. Solid State 2, 1109 (1960)].

2 Y. A. Bychkov and E. I. Rashba, Oscillatory effects and the magnetic susceptibility of carriers in inversion layers, J. Phys. C 17, 6039 (1984)

3 J. Nitta, T. Akazaki, H. Takayanagi, and T. Enoki, Gate Control of Spin-Orbit Interaction in an Inverted $I n_{0.53} G a_{0.47} A s / \operatorname{In}_{0.52} A l_{0.48}$ As Heterostructure, Phys. Rev. Lett. 78, 1335 (1997).

${ }^{4}$ Y. Sato, T. Kita, S. Gozu, and S. Yamada, Large spontaneous spin splitting in gate-controlled two-dimensional electron gases at normal $\operatorname{In}_{0.75} \mathrm{Ga}_{0.25} \mathrm{As} / \operatorname{In}_{0.75} A l_{0.25} \mathrm{As}$ heterojunctions, J. Appl. Phys. 89, 8017 (2001).

5 A. J. A. Beukman, F. K. de Vries, J. van Veen, R. Skolasinski, M. Wimmer, F. Qu, D. T. de Vries, B.-M. Nguyen, W. Yi, A. A. Kiselev, M. Sokolich, M. J. Manfra, F. Nichele, C. M. Marcus, and L. P. Kouwenhoven, Spinorbit interaction in a dual gated InAs/GaSb quantum well, arXiv: 1704.03482

6 R. I. Shekhter, O. Entin-Wohlman, and A. Aharony, Mechanically controlled Rashba spin splitters, Phys. Rev. Lett. 111, 176602 (2013).

7 R. I. Shekhter, O. Entin-Wohlman and A. Aharony, Mechanically controlled spin-selective transport, Phys. Rev. B 90, 045401 (2014).

${ }^{8}$ R. I. Shekhter, O. Entin-Wohlman, M. Jonson, and A. Aharony, Rashba spin splitting of single electrons and Cooper pairs, Phys. Rev. Lett. 116, 217001 (2016).

${ }^{9}$ Ya. M. Blanter and M. Büttiker, Shot noise in mesoscopic conductors, Phys. Rep. 336, 1 (2000).

10 A. Cottet, W. Belzig, and C. Bruder, Positive crosscorrelations in a three-terminal quantum dot with ferromagnetic contacts, Phys. Rev. Lett. 92, 206801 (2004); D. T. McClure, L. DiCarlo, Y. Zhang, H. A. Engel, C. M. Marcus, M. P. Hanson, and A. C. Gossard, Tunable Noise Cross Correlations in a Double Quantum Dot, ibid. 98, 056801 (2007).

11 Since we cannot know exactly where the two electrons "meet", we average over all the possible location of this "quantum dot".

12 As we focus on equilibrium transfers of pairs of electrons, it is assumed tacitly that the chemical potentials of the leads are identical. Under these circumstances, our calculation produces just the equilibrium Josephson current.
13 G. Dresselhaus, Spin-Orbit Coupling Effects in Zinc Blende Structures, Phys. Rev. 100, 580 (1955).

14 See, e.g., R. I. Shekhter, O. Entin-Wohlman, M. Jonson, and A. Aharony, Rashba spin-splitting of single electrons and Cooper pairs, Low Temp. Phys. 43, 303 (2017) [Fiz. Niz. Temp. 43, 368 (2017)].

15 The time derivative leads to a commutator of the particle number with the Hamiltonian; the commutator with the BCS interaction [the last term on the right-hand side of Eq. [3] vanishes in the quantum average.

16 M. Gisselfält, Parity Effects in Josephson Tunneling through a Metallic Island, Physica Scripta 54, 397 (1996).

17 The other possibility, of a dot which is initially occupied, is also of interest. We hope to pursue the spin-splitting phenomenon for this configuration in the future.

18 For simplicity, the expression for the Josephson current is presented at zero temperature; the extension to finite temperatures is quite straightforward. The subscript $L$ on the current is not needed, as at equilibrium $J_{L}=J_{R}$.

19 L. I. Glazman and K. A. Matveev, Resonant Josephson current through Kondo impurities in a tunnel barrier, JEPT Lett. 49, 659 (1989) [Pis'ma Zh. Eksp. Teor. Fiz. 49, 570 (1989)].

20 We postulate in our model that current $J_{0}$ defined by Eqs. (10) and 12 does not depend on the location of the dot.

21 We have adopted for brevity the simplified notation $W_{\sigma_{L} \sigma}^{L D}$ for $\left[W^{L D}\right]_{\sigma_{L} \sigma}$, etc.

22 Equation 15 reproduces our result in Ref. 8, valid for the Coulomb-blockade regime.

23 As explained in Ref. 8, the two terms in Eq. 15 have opposite signs due to a Josephson tunneling " $\pi$-shift" caused by electronic spin flips.

24 O. Entin-Wohlman, A. Aharony, Y. M. Galperin, V. I. Kozub, and V. Vinokur, Orbital ac spin-Hall effect in the hopping regime, Phys. Rev. Lett. 95, 086603 (2005).

25 Another source for SOI are strains, e.g., when a single flat graphene ribbon is rolled up to form a tube, see M. S. Rudner and E. I. Rashba, Spin relaxation due to deflection coupling in nanotube quantum dots, Phys. Rev. B 81, 125426 (2010); K. Flensberg and C. M. Marcus, Bends in nanotubes allow electric spin control and coupling, Phys. Rev. B 81, 195418 (2010).

${ }^{26}$ Equations 15 and 16 for $\mathcal{A}^{s}$ and $\mathcal{A}^{d}$ are valid for straight and bent weak links. For simplicity, we presented numerical results only for the simpler case of a straight link, for which $\mathcal{A}^{s}$ and $\mathcal{A}^{d}$ are given by Eq. 18 . 